\title{
Cancer gene therapy: an awkward adolescence
}

\author{
Michael M Gottesman \\ Laboratory of Cell Biology, Center for Cancer Research, National Cancer Institute, National Institutes of \\ Health, Department of Health and Human Services, Bethesda, Maryland 20892, USA.
}

\begin{abstract}
At the Eleventh International Conference on Gene Therapy of Cancer (December 12-14, 2002, San Diego, CA) progress on using gene transfer technology to treat cancer was presented. Although there is as yet no cancer gene therapy being marketed, considerable progress has been made in defining likely strategies and likely targets for gene therapy of cancer. These strategies, including viral and non-viral delivery systems, and potential targets in cancer cells linked to our developing knowledge of cancer cell biology, are reviewed in this paper. Use of gene therapy to sensitize tumors to radiation and chemotherapy is one promising area of investigation. Some of the ancillary benefits of research on cancer gene therapy, including the development of publicprivate partnerships, recruitment of laboratory scientists into clinical research, and credentialing of potential cancer cell targets for therapies other than gene therapy, are noted.

Cancer Gene Therapy (2003) 10, 501-508. doi:10.1038/sj.cgt.7700602
\end{abstract}

Keywords: vectors; apoptosis; tumor suppressors; angiogenesis; immunotherapy

$\mathrm{T}$ he first human gene transfer effort was a tumorinfiltrating lymphocyte (TIL) marking study led by Dr Steven Rosenberg of the NCI, NIH. ${ }^{1,2}$ Following a recommendation for approval by the NIH Recombinant DNA Advisory Committee (RAC), this protocol was approved by the NIH director in 1989 for the purpose of tracking lymphocytes in the immunologic treatment of melanoma and renal cell cancer. After 14 years, investigators studying gene therapy approaches to treating cancer have not yet received FDA authorization to market a gene therapy agent demonstrated to be safe and effective in the treatment of cancer. Although this track record is not too different from the other biological agents used in the treatment of disease (e.g., the development of monoclonal antibodies), questions remain about the potential of gene therapy to improve the treatment of cancer. At the Eleventh International Conference on Gene Therapy of Cancer (December 1214, 2002, San Diego, CA, USA), the progress of the field and future prospects were summarized. The conclusion from a review of the literature and the most recent advances is that the success of cancer gene therapy is closely tied to our imperfect understanding of the human cancer biology. Furthermore, the development of gene therapy directed to cancer has helped advance cancer research, and may yet lead to the development of new tools for palliation, treatment, and, hopefully, a cure.

Received February 5, 2003.

Address correspondence and reprint requests to: $\mathrm{Dr}$ Michael $M$ Gottesman, Laboratory of Cell Biology, Center for Cancer Research, NCI/NIH, Department of Health and Human Services, Building 37, Room 1A09, Bethesda, MD 20892,USA. E-mail: mgottesman@, nih.gov
Before summarizing specific issues related to the gene therapy of cancer, it is perhaps worthwhile to indicate why cancer gene therapy was launched with such fanfare 14 years ago. Cancer is a genetic disease in which individual cells demonstrate mutations in genes related to growth control and apoptosis, and have functional alterations that support their ability to invade and metastasize. ${ }^{3}$ The interaction of cancer cells with their microenvironment, including extracellular matrix, cells of the immune system, and cells necessary for induction of angiogenesis to sustain tumor growth, is a critical component of tumor growth. Thus, there are many potential targets in which introduction of new genes, and inactivation of active or defective genes, can limit or eliminate the growth of tumors. As our understanding of cancer biology and tumor-host interactions becomes more sophisticated, the number of targets grows, and the rationale for gene therapy approaches to cancer strengthens. Consequently, the field of cancer gene therapy is attracting a growing cadre of talented and creative individuals who see gene transfer as the most direct and rational way to alter specific gene functioning in both the host and tumor. There has been disappointment that some of the targets are not as easily inactivated as theory would predict, and the actual delivery of gene therapy in a completely safe and efficient manner has turned out to be a very complex task. Nonetheless, it remains clear that the rationale behind cancer gene therapy is still as strong as it was 14 years ago.

There are three major recurring themes in cancer gene therapy. The first is the design of strategies to kill or slow the growth of cancer cells (see Table 1); these can be targeted either to the cancer cells themselves, or to the host. The second is the development of vectors and delivery systems for gene transfer that are safe, efficient, 
Table 1 Strategies for cancer gene therapy

\begin{tabular}{|c|c|}
\hline Directed to tumor & Directed to host \\
\hline $\begin{array}{l}\text { Tumor suppressors } \\
\text { Dominant-negative genes } \\
\text { Induce apoptosis } \\
\text { Tumor-specific viruses } \\
\text { Tumor-specific gene } \\
\text { expression } \\
\text { Sensitization to radiation and } \\
\text { chemotherapy }\end{array}$ & $\begin{array}{l}\text { Block angiogenesis } \\
\text { Confer drug resistance on } \\
\text { sensitive tissues, } \\
\text { especially bone marrow } \\
\text { Immunotherapy } \\
\text { Identify and introduce } \\
\text { tumor antigens } \\
\text { Introduce cytokines } \\
\text { Engineer APCs }\end{array}$ \\
\hline
\end{tabular}

and, to the extent possible, targeted. The third major theme is the translation of preclinical studies into clinical protocols and trials with which to test the safety and efficacy of cancer gene therapy.

\section{Strategies for gene therapy of cancer}

As noted above, our success in developing strategies for gene therapy of cancer is closely tied to understanding of the biology of human cancer. As our understanding of tumor biology has advanced, the strategies employing gene transfer to treat cancer have become increasingly efficient. I will first summarize the general approaches for targeting cancer cells themselves, and then describe approaches to altering host responses that facilitate the development of cancer.

\section{Strategies directed to the cancer cells}

The most obvious way to target growth regulation in cancer cells is to introduce tumor suppressors that may be inactivated in specific tumors. Since the most commonly inactivated tumor suppressor in cancer cells is p53, this has become an obvious first target for cancer gene therapy involving tumor suppressors. A number of different viral vectors expressing p53 have been constructed, especially using adenovirus backbones. These vectors have been shown to increase p53 levels in cultured p53-defective cancer cells resulting in cessation of cell growth or induction of apoptosis in synergy with chemotherapeutic agents such as cisplatin. ${ }^{4,5}$ This approach has entered clinical trials with an adenovirus vector expressing p53 called "Advexin" by John Nemunaitis and colleagues (for a review, see Nemunaitis $^{6}$ ) in treating squamous cell carcinoma of the head and neck (SCCHN).

Other tumor suppressors have been introduced into cancer cells by gene transfer including $\mathrm{Rb}^{7}$ and mda-7 (melanocyte differentiation associated antigen-7). The latter appears to suppress tumor growth and induce apoptosis and has recently been shown to be identical to interleukin-24, which is a Th1-specific cytokine that induces the immune system resulting in antitumor and proapoptotic responses. ${ }^{8,9}$ Efforts to identify additional tumor suppressors, particularly in lung cancer, which has proved relatively intractable to more standard therapies, have resulted in the isolation of three genes that map to the region of loss of heterozygosity $(\mathrm{LOH})$ on chromosome $3 \mathrm{p}$ identified by comparative genomic hybridization (CGH). ${ }^{10}$ These genes, termed Fus1, 101F6, and NPRL2, whose expression is more potent than p53 in inhibiting tumor growth in vitro, are currently being characterized. In a similar strategy, expression of dominant-negative oncogenes and dominant-negative versions of other growth-promoting genes can be used to inactivate the function of important oncogenes in cancer cells. ${ }^{1-13}$

Induction of apoptosis, which is relatively defective in most tumor cells, is another possible strategy to kill cancer cells, either directly, or in combination with modalities such as radiation and chemotherapy, which are capable of inducing apoptosis. John Reed ${ }^{14}$ has reviewed the three common pathways for induction of apoptosis: the TNF, TRAIL, Fas pathway; the cytotoxic T-cell pathway through perforin; and the mitochondrial pathway through BAX (stimulates apoptosis) and BCL-2 (inhibits apoptosis). ${ }^{15}$ These pathways work through a common pathway involving caspase 3 , and there are various cellular inhibitors of the common apoptotic pathway, termed IAPs. The three unique pathways, and the common pathway, including its inhibitors, are all potential targets for anticancer gene therapy or drug therapy. Strategies targeted to BCL-2 (antisense, siRNA), increase of BAX and its cogeners, and inhibition of the IAPs are all potential approaches.

One of the most exciting new kinds of cancer gene therapy is the use of viruses that replicate only in tumor cells, also known as oncolytic viruses. This work began with the discovery that adenovirus lacking E1B would not grow in normal cells, but would grow in cells lacking p53, a common characteristic of cancer cells. The E1B deleted adenovirus, ONYX-015 $5^{16-18}$ has been successfully used as an adjunct to the local control of SCCHN, and is currently under evaluation for other cancers. Newcastle Disease Virus, a virus of birds, can replicate in human cancer cells, but only very poorly in normal human tissues. Hence, it, too, is undergoing clinical trials. ${ }^{19}$ The development of replication-competent retroviruses (RCR), barring an unacceptably high incidence of insertional mutagenesis events resulting in cancer (see Discussion below of serious adverse events associated with retroviral gene therapy), might also prove useful for infection and spread in tumors with a sizeable population of dividing cells, since all retroviruses, with the exception of the lentiviruses, require cell division to complete their life cycle of reverse transcription and integration into the genome. The objective in this instance is to target tumors surrounded by nondividing normal tissue, such as gliomas in the brain, and after the virus has spread throughout the tumor, to use a suicide selection gene to kill the infected tumor cells. ${ }^{20}$ One serious concern is that RCR could enter the circulation and infect dividing cells at a distance from the tumor. The presence of a suicide gene in a retroviral vector confers some measure of protection, which is not complete, against inadvertent insertional mutagenesis events that activate oncogenes. Suicide 
genes $^{21}$ allow the expression of enzymes that can convert nontoxic prodrugs to active cytotoxic drugs, and include the Herpes simplex thymidine kinase gene that converts ganciclovir to ganciclovir phosphate, and the cytidine deaminase gene that converts 5-fluorocytidine (5-FC) to 5-fluorouracil (5-FU).

For viral vectors that are introduced into the circulation, it is essential that there be targeting of the virus (see below) or targeting of gene expression to the tumor. A number of systems have been described which are quite specific in targeting expression of suicide genes or tumor suppressors to specific cancers, such as vectors carrying pancreatic cancer-specific ${ }^{22,23}$ and prostate cancer-specific $^{24,25}$ transcription units.

As noted above, a very powerful tool for treating cancer is the sensitization of cancer cells to treatments such as radiation and chemotherapy, which are known to be effective cancer treatment in their own right. In the case of radiation, local control of tumors can be achieved by combining proapoptotic regimens (expression of BAX, or TNF-alpha) with radiation. ${ }^{26}$ In addition, combinations of traditional chemotherapy with expression of tumor suppressors such as mda-7 may prove useful in enhancing tumor kill in tumors to which the suppressors can be targeted by direct injection.

\section{Cancer gene therapy directed to the host}

As already indicated, cancer cells tend to develop resistance to anticancer agents, but host cells whose function is essential for tumor development might prove to be better targets because they are less likely to mutate. Three general strategies have been tested: targeting the tumor angiogenic pathway; protecting normal tissues, especially bone marrow, from the toxic effects of chemotherapy; and modifying the immune system. Angiogenesis can be inhibited by constitutive expression of inhibitors of angiogenesis such as angiostatin, endostatin, and pigment-epithelial derived factor (PEDF). As reported at the meeting by Patrick $\mathrm{Lu}$ (Intradigm Corporation), VEGF and the VEGF receptor can be targeted with antisense or siRNA. These approaches require relatively long-term treatment, at least until regression or apoptosis of tumor cells deprived of their vasculature.

Much is known about the genes that contribute to resistance to chemotherapy in cancer. Some of these genes, including the ATP-binding cassette transporters MDR1 and MRP1 that act as energy-dependent drug efflux pumps for many natural product anticancer drugs, ${ }^{27}$ and a mutant form of the enzyme dihydrofolate reductase (DHFR), which confers resistance to methotrexate, ${ }^{28}$ have been shown to confer resistance on normal human tissues such as bone marrow, and to protect transgenic animals from the toxic effects of these drugs. Based on these studies, attempts to protect normal marrow in patients undergoing bone marrow transplantation have been made using retroviral vectors carrying the MDR1 gene. ${ }^{29}$ Although some selective advantage has been attributed to cells carrying this gene, complete

protection of bone marrow has not been possible because of the relatively low frequency of transduction.

The immune system has proved to be both a common and potentially useful target in cancer gene therapy. Therapies have been targeted to immune effector cells including dendritic (antigen processing), natural killer (NK) cells, and CD8 cells. Introduction into these cells of cytokines or exposure to cloned antigens that amplify their immune reactivity are under development. ${ }^{30,31}$ Cancer vaccines based on modification of the tumor cells themselves by cytokines such as GM-CSF, which stimulate the immune system, have been shown to be effective in preclinical models ${ }^{32}$ and have entered clinical trials.

\section{Vectors and gene delivery systems}

The search for ways to introduce genes into cancer cells led to many advances in viral and nonviral vector development (Table 2). The viral vector most commonly used in cancer gene therapy is the adenoviral vector because of the ease with which high titer lysates, free of wild-type virus, can be prepared, and this virus' broad spectrum of tissue tropism in humans. ${ }^{33}$ Also, it is possible to alter the surface fiber proteins to change tissue tropism, ${ }^{34}$ and since quite a bit is known about the regulation of adenoviral gene expression and the function of adenoviral genes, it has been possible to substitute tissue-specific promoters to allow cancer-specific gene expression. One recent advance in changing the tropism of adenovirus is the introduction of a biotin-binding protein into the major fiber protein that can be used to attach via a biotin-avidin system-specific ligands for cell targeting (Michael Barry, Baylor College of Medicine). The disadvantages of using adenovirus for gene therapy are well known: high immunogenicity, proinflammatory effects, and lack of stable integration. The death of a research participant enrolled in a gene therapy clinical trial for ornithine transcarbamylase deficiency ${ }^{35}$ that occurred following intrahepatic artery administration has been largely attributed to an adenovirus-induced systemic immune response with coma and respiratory failure; ${ }^{36}$ however, systemic responses to adenovirus including fever, hypotension, and coagulopathies are not uncommon. ${ }^{37}$

As noted, retroviruses continue to be used as possible gene delivery vehicles, although recent events raise concerns about insertional mutagenesis. Although titers

Table 2 Vectors and delivery systems

Viral systems

Adenovirus

Retroviruses, especially lentivirus HSV

SV40

Newcastle disease virus Others
Nonviral systems

Lipofection
Bacteria
Nucleotides
Antisense
Ribozymes
siRNA


are much lower than adenovirus, the use of RCRs may prove a valuable adjunct allowing local spread of retroviruses introduced into a tumor. A downside of this approach is the inability to deliver retroviruses systemically, but this can be overcome using hybrid delivery systems in which the retroviral gene is first introduced using a gutted vector such as adenovirus. ${ }^{20}$ Lentiviruses, which do not require a dividing cell to integrate into the genome, are also under development for gene therapy. ${ }^{38}$ As they are derived from HIV, particular care must be taken to package them in cell lines in which recombination to produce a replication-competent virus cannot take place. $^{39}$ For retroviruses in general, their obligate integration into the genome, while facilitating stable, long-term expression, is also mutagenic. In a clinical trial for X-linked severe combined immunodeficiency (SCID) using a retroviral vector, ${ }^{40}$ at least two of the 11 children enrolled have developed leukemia secondary to retroviral insertion. One of these cases has been reported to date $^{41}$ and the NIH has provided information about the second case (see http://www4.od.nih.gov/oba/RAC/ X-SCID.htm). This raises serious concerns about the use of retroviral vectors for gene therapy in patients likely to survive their treatment.

Herpes simplex virus is receiving increasing attention because of its ability to replicate and kill cells very efficiently. When appropriately engineered, replication competent herpesviruses incapable of growth in normal human tissues can be shown to produce cytopathic effects and cell death in cancers. Herpesviruses may prove particularly useful for treatment of gliomas in the central nervous system because of their known tropism for nervous tissue. ${ }^{42,43}$

Although initially thought to be limited in ability to package DNAs, SV40-based vectors also show promise for gene transfer. The SV40 major capsid protein, VP1, can be used in vitro to package any supercoiled DNA up to $18 \mathrm{kDa}$ in size. These DNAs are introduced at high efficiency into most cell types, especially those expressing MHC Class I, which is thought to be the major receptor for SV40. Expression levels are relatively low and transient, but for some purposes such as targeted introduction of toxin genes or siRNA, this may prove to be a useful vector system because of its ease of use, lack of antigenicity, and apparent safety (no integration, no inflammation). ${ }^{44} \mathrm{~A}$ number of other viruses have been used over the past several years for gene therapy, including Newcastle Disease Virus (see above), vaccinia virus for tumor antigen expression, ${ }^{45}$ and other orthopoxviruses, such as canarypox. ${ }^{46}$

For nonviral delivery systems, most progress has been made using variations of lipofection. In this approach, DNA is associated with lipids and can enter cells either by endocytosis or by fusion with the cell membrane. One current system that appears to combine high efficiency of entry into cells via membrane fusion (avoiding the endocytic degradation pathway), with lack of toxicity and ability to be used intravenously, is the DOTAP:cholesterol system. ${ }^{47}$ Modifications are in progress to coat the outside of these vesicles to prevent trapping in the lungs, and to allow targeting to specific tissues. For aerosol delivery of DNA into the lungs, polyethyleneimine (PEI) has been used with some success. ${ }^{48}$ This may be a desirable approach to the treatment of lung cancer.

Bacteria are capable of invading tissues and can be used for the delivery of genes to cancer cells. John Nemunaitis (US Oncology) is spearheading a trial using Salmonella carrying cytidine deaminase genes as a possible prodrug activating system for patients with advanced cancer. ${ }^{49}$

Finally, therapy with nucleotides, either by direct treatment of cells by systemic administration, or carried on various liposomes, has been a goal of gene therapy investigators for many years. Strategies that employ antisense RNA, ribozymes, or siRNA are under development. Progress in chemical modifications of polynucleotides to improve stability in vivo includes the clinical development of phosphoroamidate morphilino (PMO) nucleotides for antisense therapy. ${ }^{50}$ Some large-scale clinical trials with antisense RNA will be completed in the next year to determine whether these approaches are effective in certain cancers in which clear molecular targets can be defined, such as leukemias and lymphomas.

\section{Trends in gene cancer clinical trials}

The NIH Recombinant DNA Advisory Committee has been overseeing human gene therapy since the first gene transfer marking trial in 1988. Data assembled by the RAC over these years gives a good sense of the changes in trends in targets for cancer gene therapy and in vectors used for clinical trials. Figure 1 shows that the majority of gene therapy trials have been directed at cancer (360 out of a total of 570 analyzed). Of these cancer gene therapy trials, fully half have been directed against five cancers: melanoma, prostate, ovary, squamous cell carcinoma of the head and neck, and leukemia (Fig 2). These were chosen both because of specific opportunities to treat them based on the strategies outlined in Table 1, but also because in some cases (cancers of the prostate, ovary, $\mathrm{SCCHN}$ ), local control is a primary goal of cancer therapy. Trends in the use of vectors for gene delivery in
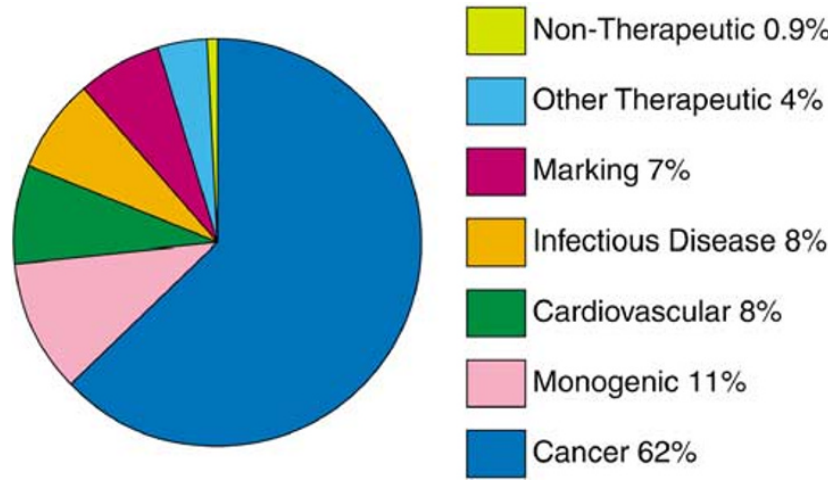

Figure 1 Gene transfer protocols by application. There were 570 total protocols. Data provided courtesy of Amy Patterson, Director, Office of Biotechnology Activities, NIH, DHHS. 

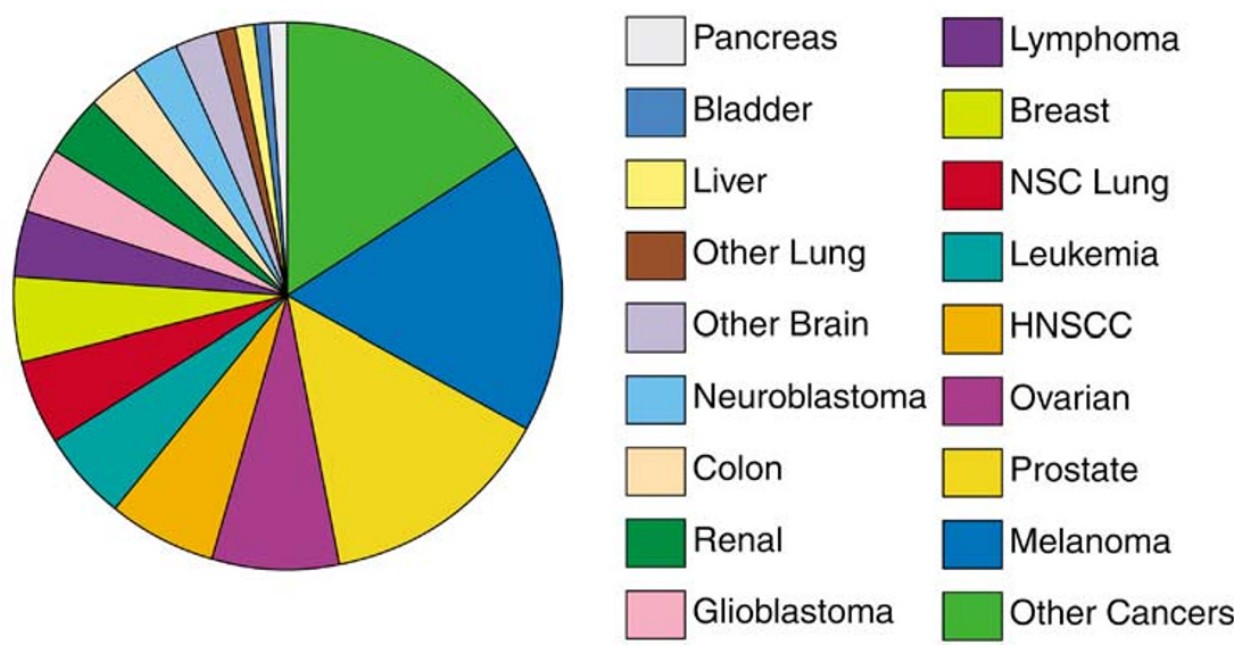

Figure 2 Cancer gene transfer protocols by application. There were 360 total cancer protocols. Data provided courtesy of Amy Patterson, Director, Office of Biotechnology Activities, NIH, DHHS.

cancer gene therapy (Fig 3) show an equal mix of retrovirus, adenovirus, and plasmids (with poxviruses used for vaccine delivery), but indicate clear temporal trends with decreasing use of retroviral vectors and increasing use of adenovirus vectors and plasmids (Fig 4). Although there is an apparent increase in relative use of retroviral vectors in the first month of 2003, this percentage is based on a very low number of trials and does not yet reflect the impact of the serious adverse events that occurred in the X-linked SCID trials.

\section{Adverse events in the gene therapy of cancer}

Three widely publicized serious adverse events, including one death, ${ }^{36}$ has occurred in gene therapy of monogenic diseases and have been attributed directly to the gene
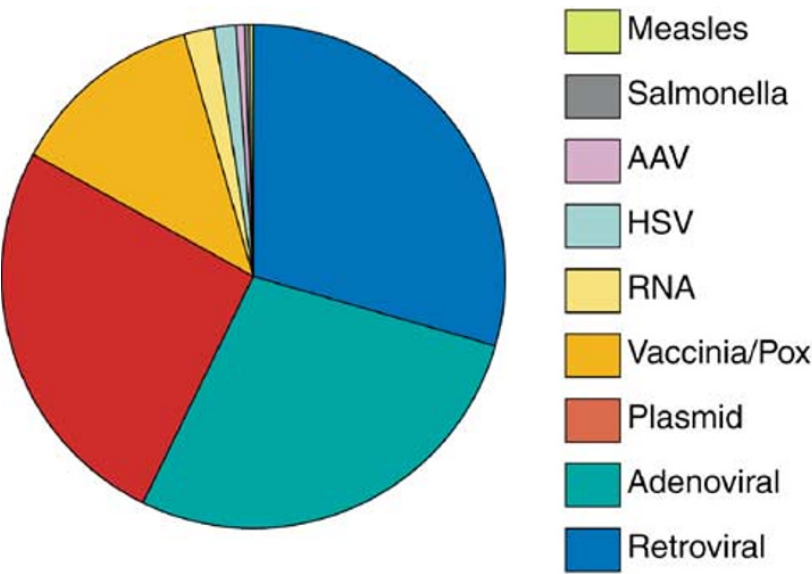

Figure 3 Vector usage in cancer gene transfer protocols. Data provided courtesy of Amy Patterson, Director, Office of Biotechnology Activities, NIH, DHHS. therapy intervention. The definitive attribution of adverse events in cancer gene therapy clinical trials to the gene therapy product or to the underlying disease and/or concomittant interventions such as chemotherapy or surgery, however, is often difficult. In part, this is because of the fact that, to date, patients who are subjects for cancer gene therapy trials have a fatal, generally rapidly progressive disease, and it is often difficult to discern the negative effects of gene therapy against a background of pathology associated with cancer. Many cancer gene therapy protocols are written so that patients whose underlying disease progresses while on cancer gene therapy are removed from the trials; thus, if death occurs, it usually occurs off-protocol. A new emphasis on reporting serious adverse events associated with gene therapy trials that are unanticipated based on either the disease progression or the known side effects of the treatment protocols has revealed occasional severe inflammatory reactions associated with viral vectors and mild to moderate coagulopathies.

To facilitate reporting of serious, unanticipated adverse events, the FDA and the NIH have collaborated to harmonize reporting requirements. In addition, the NIH has worked with the FDA to develop the Genetic Modification Clinical Research Information System (GeMCRIS), a Web-accessible system for collecting and analyzing data about gene therapy trials. GeMCRIS will greatly facilitate our understanding of the science and safety of this field of research by permitting comparative analyses of data and cross-tabulations across all trials in the system. An important feature of GeMCRIS will be broad-public access — by scientists and patients alike — to these aggregated data, while protecting confidential commercial information through the use of firewalls and other security measures. GeMCRIS is currently being beta-tested at several major medical centers in the US and is due for release in the spring of 2003. 

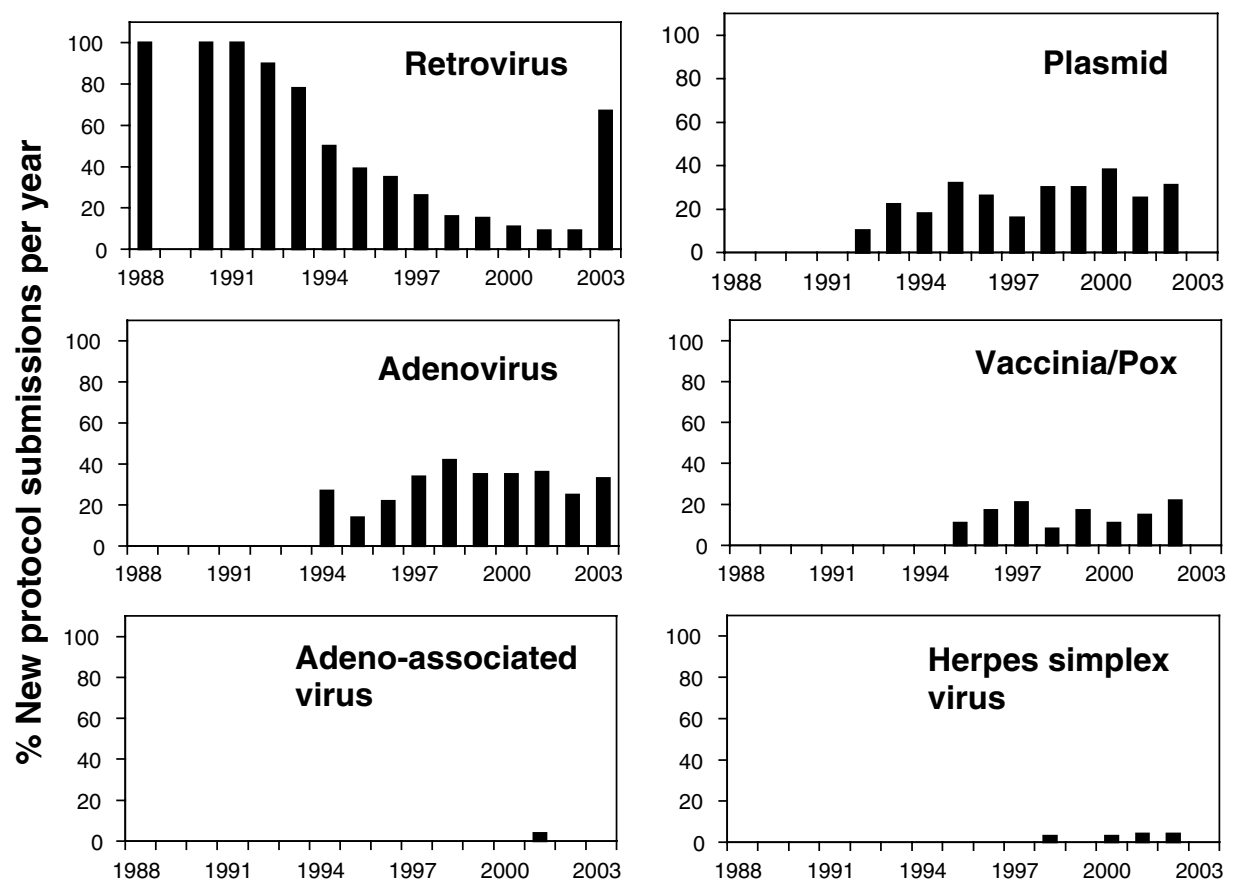

Figure 4 Trends in vector usage in cancer protocols submitted 1988-2003. Data provided courtesy of Amy Patterson, Director, Office of Biotechnology Acitivities, NIH, DHHS.

\section{Final perspectives}

It is clear that gene therapy of cancer is a very difficult undertaking. As researchers, we do not yet know enough about the biology of cancer, the host response to cancer, how the immune system works, or how to develop safe, efficient, and targeted vectors for gene delivery. Despite these reservations, some progress has been made in building infrastructure to study gene therapy; partnerships among industry, academia, and the government have resulted in substantial investments in a field that is yet to produce a cure or a marketable product. Furthermore, the concepts behind cancer gene therapy have encouraged investigators, both with medical and laboratory training, to think about and become involved in creative new approaches to the treatment of cancer.

Even for the pessimist, at a minimum the field of cancer gene therapy has provided proof of concept for the targeting of many new genes and gene products in cancer cells. Although these may not be targeted in the future with gene therapy, they may well be credentialed by the process of gene therapy of cancer, and become credible targets for anticancer drug development. For the more optimistic, the immediate future of cancer gene therapy would suggest increasing success in using cancer gene therapy as adjunct therapy in local control of many cancers. Although perhaps not capable of effecting cures, cancer gene therapy may prove useful in the incremental improvement of tools currently in use to treat cancer. For the most optimistic, the "big hit" is just around the corner; perhaps with an oncolytic virus or a replication- competent retrovirus carrying a suicide gene, a cancer will be eradicated.

\section{Acknowledgments}

I am grateful to George Leiman for editorial assistance and to Amy Patterson for comments on the manuscript.

\section{References}

1. Aebersold P, Kasid A, Rosenberg SA. Selection of genemarked tumor infiltrating lymphocytes from post-treatment biopsies: a case study. Hum Gene Ther. 1990;1:373-384.

2. Cai Q, Rubin JT, Lotze MT. Genetically marking human cells - results of the first clinical gene transfer studies. Cancer Gene Ther. 1995;2:125-136.

3. Hanahan D, Weinberg RA. The hallmarks of cancer. Cell. 2000;100:57-70.

4. Yen $\mathrm{N}$, Ioannides $\mathrm{CG}, \mathrm{Xu} \mathrm{K}$, et al. Cellular and humoral immune responses to adenovirus and p53 protein antigens in patients following intratumoral injection of anadenovirus vector expressing wild-type p53 (Ad-p53). Cancer Gene Ther. 2000;7:530-536.

5. Horio Y, Hasegawa Y, Sekido Y, Takahasi M, Roth JA, Shimokata K. Synergistic effects of adenovirus expressing wild-type p53 on chemosensitivity of nonsmall cell lung cancer cells. Cancer Gene Ther. 2000; 7:537544.

6. Nemunaitis J. Live viruses in cancer treatment. Oncology. 2002;16:1483-1492. 
7. Demers GW, Harris MP, Wen SF, Engler H, Nielsen LL, Maneval DC. A recombinant adenoviral vector expressing full-length human retinoblastoma susceptibility gene inhibits human tumor cell growth. Cancer Gene Ther. 1998;5:207-214.

8. Sauane M, Gopalkrishnan RV, Sarkar D, et al. MDA-7/IL24: novel cancer growth suppressing and apoptosis inducing cytokine. Cytokine Growth Factor Rev. 2003;14:35-51.

9. Caudell EG, Mumm JB, Poindexter N, et al. The protein product of the tumor suppressor gene, melanoma differentiation-associated gene 7 , exhibits immunostimulatory activity and is designated IL-24. $J$ Immunol. 2002;168:6041-6046.

10. Ji L, Nishizaki M, Gao B, et al. Expression of several genes in the human chromosome 3p21.3 homozygous deletion region by an adenovirus vector results in tumor suppressor activities in vitro and in vivo. Cancer Res. 2002;62:27152720.

11. Heinicke T, Radziwill G, Nawrath M, Rommel C, Pavlovic J, Moelling K. Retroviral gene transfer of dominant negative raf-1 mutants suppresses Ha-ras-induced transformation and delays tumor formation. Cancer Gene Ther. 2000;7:697-706.

12. Lee CT, Park KH, Adachi Y, et al. Recombinant adenoviruses expressing dominant negative insulin-like growth factor-I receptor demonstrate antitumor effects on lung cancer. Cancer Gene Ther. 2003;10:57-63.

13. Benovich M, Olive M, Reed E, O'Connell B, Vinson C. Adenoviral delivery of A-FOS, an AP-1 dominant negative, selectively inhibits drug resistance in two human cancer cell lines. Cancer Gene Ther. 2002;9:62-70.

14. Reed JC. Apoptosis-based therapies. Nat Rev Drug Discov. 2002;1:111-121

15. Roth W, Reed JC. Apoptosis and cancer: When BAX is TRAILing away. Nat Med. 2002;8:216-218.

16. Heise CC, Williams A, Olesch J, Kirn DH. Efficacy of a replication-competent adenovirus (ONYX-015) following intratumoral injection: intratumoral spread and distribution effects. Cancer Gene Ther. 1999;6:499-504.

17. Biederer C, Ries S, Brandts $\mathrm{CH}$, McCormick F. Replication-selective viruses for cancer therapy. $\mathrm{J}$ Mol Med. 2002;80:163-175.

18. Yoon TK, Shichinohe T, Laquerre S, Kasahara N. Selectively replicating adenoviruses for oncolytic therapy. Curr Cancer Drug Targets. 2001;1:85-107.

19. Pecora AL, Rizvi N, Cohen Gi, et al. Phase I trial of intravenous administration of PV701, an oncolytic virus, in patients with advanced solid cancers. $J$ Clin Oncol. 2002;20:2251-2266.

20. Soifer H, Higo C, Logg CR, et al. A novel, helperdependent, adenovirus-retrovirus hybrid vector: stable transduction by a two-stage mechanism. Mol Ther. 2002;5:599-608.

21. Moolten FL. Drug sensitivity ("suicide") genes for selective cancer chemotherapy. Cancer Gene Ther. 1994; 1:279-287.

22. Yoshida $\mathrm{Y}$, Tomizawa M, Bahar R, et al. A promoter region of midkine gene can activate transcription of an exogenous suicide gene in human pancreatic cancer. Anticancer Res. 2002;22:117-120

23. Wesseling JG, Yamamoto M, Adachi Y, et al. Midkine and cyclooxygenase-2 promoters are promising for adenoviral vector gene delivery of pancreatic carcinoma. Cancer Gene Ther. 2001;8:990-996.
24. DeWeese TL, van der Poel H, Li S, et al. A phase I trial of CV706, a replication-competent, PSA selective oncolytic adenovirus, for the treatment of locally recurrent prostate cancer following radiation. Cancer Res. 2001;61: 7464-7472.

25. Marintiello-Wilks R, Tsatralis T, Russell P, et al. Transcription-targeted gene therapy for androgen-independent prostate cancer. Cancer Gene Ther. 2002;9:443-452.

26. Rasmussen H, Rasmussen C, Lempicki M, et al. TNFerade Biologic: preclinical toxicology of a novel adenovector with a radiation-inducible promoter, carrying the human tumor necrosis factor alpha gene. Cancer Gene Ther. 2002;9:951957.

27. Gottesman MM, Fojo T, Bates SE. Multidrug resistance in cancer: role of ATP-dependent transporters. Nat Rev Cancer. 2002;2:48-58.

28. Braun SE, McIvor RS, Davidson AS, et al. Retrovirally mediated gene transfer of $\operatorname{Arg} 22$ and Tyr22 forms of dihydrofolate reductase into the hematopoietic cell line K562: a comparison of methotrexate resistance. Cancer Gene Ther. 1997;4:26-32.

29. Cowan KH, Moscow JA, Huang H, et al. Paclitaxel chemotherapy after autologous stem-cell transplantation and engraftment of hematopoietic cells transduced with a retrovirus containing the multidrug resistance complementary DNA (MDR1) in metastatic breast cancer patients. Clin Cancer Res. 1999;5:1619-1628.

30. Dalal RM, Lotze MT. Immunotherapy of metastasis. Surg Oncol Clin N Am. 2001;10:433-447.

31. Reyes-Sandoval A, Ertl HC. DNA vaccines. Curr Mol Med. 2001;1:217-243.

32. Wang L, Qi X, Sun Y, Liang L, Ju D. Adenovirus-mediated combined P16 gene and GM-CSF gene therapy for the treatment of established tumor and induction of antitumor immunity. Cancer Gene Ther. 2002;9:819-824.

33. Bauerschmitz GJ, Barker SD, Hemminki A. Adenoviral gene therapy for cancer: from vectors to targeted and replication competent agents (Review). Int $J$ Oncol. 2002;21:1161-1174.

34. Haviv YS, Blackwell JL, Kanerva A, et al. Adenoviral gene therapy for renal cancer requires retargeting to alternative cellular receptors. Cancer Res. 2002;62:4273-4281.

35. Raper SE, Yudkoff M, Chirmule N, et al. A pilot study of in vivo liver-directed gene transfer with an adenoviral vector in partial ornithine transcarbamylase deficiency. Hum Gene Ther. 2002;13:163-175.

36. Marshall E. Gene therapy death prompts review of adenovirus vector. Science. 1999;286:2244-2245.

37. Anderson WF (ed.). NIH report: assessment of adenoviral vector safety and toxicity: report of the National Institutes of Health Recombinant DNA Advisory Committee. Cancer Gene Ther. 2002;13:3-13.

38. Shichinohe T, Bochner BH, Mizutani K, et al. Development of lentiviral vectors for antiangiogenic gene delivery. Cancer Gene Ther. 2001;8:879-889.

39. Kafri T, van Praag H, Ouyang L, Gage FH, Verma IM. A packaging cell line for lentivirus vectors. $J$ Virol. 1999;73:576-584.

40. Fischer A, Hacein-Bey S, Cavazzana-Calvo M. Gene therapy of severe combined immunodeficiencies. Nat Rev Immunol. 2002;2:615-621.

41. Hacein-Bey-Abina S, von Kalle C, Schmidt M, et al. A serious adverse event after successful gene therapy for Xlinked severe combined immunodeficiency. $N$ Engl $J$ Med. 2003;348:255-256. 
42. Barnett FH, Rainov NG, Ikeda K, et al. Selective delivery of herpes virus vectors to experimental brain tumors using RMP-7. Cancer Gene Ther. 1999;6:14-20.

43. Lilley CE, Branston RH, Coffin RS. Herpes simplex virus vectors for the nervous system. Curr Gene Ther. 2001;1:339358.

44. Kimchi-Sarfaty C, Ben-Nun-Shaul O, Rund D, Oppenheim A, Gottesman MM. In vitro-packaged SV40 pseudovirions as highly efficient vectors for gene transfer. Hum Gene Ther. 2002;13:299-310.

45. Paul S, Regulier E, Rooke R, et al. Tumor gene therapy by MVA-mediated expression of T-cell-stimulating antibodies. Cancer Gene Ther. 2002;9:470-477.

46. Odin L, Favrot M, Poujol D, et al. Canarypox virus expressing wild type p53 for gene therapy in murine tumors mutated in p53. Cancer Gene Ther. 2001;8: 87-98.

47. Templeton NS, Lasic DD, Frederik PM, Strey HH, Roberts DD, Pavlakis GN. Improved DNA : liposome complexes for increased systemic delivery and gene expression. Nat Biotechnol. 1997;15:647-652.

48. Orson FM, Kinsey BM, Bhogal BS, Song L, Densmore CL, Barry MA. Targeted delivery of expression plasmids to the lung via macroaggregated polyethylenimine-albumin conjugates. Methods Mol Med. 2003;75:575-590.

49. Cunningham C, Nemunaitis J. A phase I trial of genetically modified Salmonella typhimurium expressing cytosine deaminase (TAPET-CD, VNP20029) administered by intratumoral injection in combination with 5-fluorocytosine for patients with advanced or metastatic cancer. Hum Gene Ther. 2001;10:1594-1596.

50. Devi GR. Prostate cancer; status of current treatments and emerging antisense-based therapies. Curr Opin Mol Ther. 2002;4:138-148. 